ORIGINAL ARTICLE

\title{
Transformed dermatofibrosarcoma protuberans: a clinicopathological study of eight cases
}

\author{
Z Szollosi, Z Nemes
}

J Clin Pathol 2005;58:751-756. doi: 10.1136/icp.2004.019349

See end of article for authors' affiliations

....................

Correspondence to:

Dr Z Szollosi, Department of Pathology, University of Debrecen, Medical and Health Science Centre, Debrecen, $\mathrm{H}-4012$

Hungary; szollosi@jaguar. dote.hu

Accepted for publication 21 December 2004

\begin{abstract}
Background: Fibrosarcomatous (FS) or malignant fibrous histiocytomatous (MFH) transformation of dermatofibrosarcoma protuberans (DFSP) is a rare, but well known, entity. DFSPs with sarcomatous areas have questionable biological behaviour. Several studies suggest that they have a higher risk for local recurrence and distant metastases than ordinary DFSPs. One recent study described no difference in the behaviour of conventional and transformed DFSP.

Aims: To investigate the biological behaviour of a series of transformed DFSPs.

Methods: Eight transformed DFSPs were analysed clinicopathologically. Follow up ranged from four to 36 years.

Results: The tumours involved the trunk (six cases) and lower extremity (two cases) and measured 3.5$8 \mathrm{~cm}$ (median, 4). Sarcomatous change presented de novo in all cases. The type of sarcomatous change was FS (five cases) and MFH (three cases). The estimated proportion of sarcomatous area in the tumour was $25-70 \%$ (median, 43.37\%). Mitotic counts ranged from nine to 16 mitotic figures/10 high power fields in the FS and MFH areas (median, 12), and from one to three in the DFSP areas. Six patients were treated by wide local excision with histopathologically negative margins and two were treated by simple surgical excision with positive margins. Three patients developed recurrences and one developed metastasis during follow up. Of those treated by wide local excision, one developed recurrence. All tumours expressed CD34 in the DFSP component, but only three in the sarcomatous area.

Conclusions: Although DFSP containing sarcoma may be a more aggressive tumour, its behaviour can be influenced by surgical treatment.
\end{abstract}

"There seems to be a poor correlation between the size of the tumour and the recurrence rate, but the completeness of excision and the distance of margins from the tumour have been reported to affect recurrence"

DFSP is characterised clinically by locally aggressive growth and a high rate of local recurrence, but distant metastases $(1-4 \%)$ and tumour related deaths are very rare. ${ }^{1}$ Recurrence is common in patients with DFSP and the literature suggests an incidence of $20-50 \% .^{2}{ }^{3}$ There seems to be a poor correlation between the size of the tumour and the recurrence rate, but the completeness of excision and the distance of margins from the tumour have been reported to affect it. ${ }^{1}$ Several reports suggest that excision with at least $2 \mathrm{~cm}$ of free margin (so called wide local excision) reduces the relapse rate significantly. ${ }^{2}$

DFSP is morphologically heterogeneous; several rare variants have been described that are important to recognise to avoid misdiagnosis with more aggressive tumours. Most of the variants of DFSP are not associated with significant differences in clinical behaviour. ${ }^{45}$
A small number of cases of DFSP contain areas of fibrosarcoma (FS) or, more rarely, malignant fibrous histiocytoma (MFH). During the past two decades, several case reports and small series of DFSP with FS-like or MFHlike areas have been published. These variants of DFSP have questionable prognostic relevance..$^{6-16}$

Our study investigates the biological behaviour of transformed DFSP by analysing a series of patients with longterm follow up.

\section{MATERIALS AND METHODS}

Eighty seven consecutive cases of DFSP were retrieved from the routine histological files of the department of pathology, University of Debrecen, Debrecen, Hungary. Formalin fixed, paraffin wax embedded material was available for evaluation. Paraffin wax embedded sections from all cases were processed routinely and stained with haematoxylin and eosin. The haematoxylin and eosin stained slides of each specimen were reviewed to identify sarcomatous changes. The following diagnostic criteria were used: (1) the presence of classic morphological features of DFSP and (2) the presence of sarcomatous change in at least $5 \%$ of the lesion. FS was defined as a mitotically active cellular area composed of spindle cells with FS change/FS transformation arranged in a fascicular pattern. MFH transformation was defined as a mitotically active cellular area composed of pleomorphic, undifferentiated cells. Eight cases that fulfilled these criteria were included in our study.

In these eight cases the following features were recorded: tumour size; area of sarcomatous transformation; estimated

Abbreviations: DFSP, dermatofibrosarcoma protuberans; FS, fibrosarcoma; HPF, high power field; MF, mitotic figure; MFH, malignant fibrous histiocytoma 
Table 1 Data on patients with DFSP-FS and DFSP-MFH

\begin{tabular}{|c|c|c|c|c|c|c|c|c|c|c|}
\hline Case & Age/sex & Site & $\begin{array}{l}\text { Tumour } \\
\text { size }(\mathrm{cm})\end{array}$ & $\begin{array}{l}\text { Type of } \\
\text { sarcoma }\end{array}$ & $\begin{array}{l}\text { Occurrence } \\
\text { of sarcoma }\end{array}$ & $\begin{array}{l}\text { Surgical } \\
\text { margins }\end{array}$ & Treatment & Recurrences & Metastases & Follow up \\
\hline 1 & $26 / F$ & Back & $4 \times 3 \times 2.5$ & FS & De novo & Negative & WLE & - & - & NED; 36 years \\
\hline 2 & $36 / F$ & Chest wall & $4.5 \times 3 \times 3$ & FS & De novo & Negative & WLE & - & - & NED; 20 years \\
\hline 3 & $56 / M$ & Leg & $6 \times 4 \times 4$ & MFH & De novo & Positive & ILE, irrad, PC & 2 & - & DUC; 17 yrs \\
\hline 4 & $41 / F$ & Trunk & $4 \times 3 \times 3$ & FS & De novo & Negative & WLE & 1 & - & NED; 6 years \\
\hline 5 & $39 / F$ & Abdominal wall & $4 \times 2.5 \times 2$ & FS & De novo & Negative & WLE & - & - & NED; 5 years \\
\hline 6 & $62 / M$ & Trunk & $8 \times 3 \times 6$ & MFH & De novo & Positive & ILE, irrad & 3 & + & DUC; 13 years \\
\hline 7 & $28 / F$ & Trunk & $5 \times 3.5 \times 3$ & FS & De novo & Negative & WLE & - & - & NED; 4 years \\
\hline 8 & $37 / F$ & Leg & $3.5 \times 3 \times 3$ & MFH & De novo & Negative & WLE & - & - & NED; 5 years \\
\hline
\end{tabular}

DFSP, dermatofibrosarcoma protuberans; DUC, died of unrelated cause; FS, fibrosarcoma; ILE, incomplete local excision; irrad, irradiation; MFH, malignant fibrous histiocytoma; NED, no evidence of disease; PC, postoperative chemotherapy; WLE, wide local excision.

percentage of DFSP and sarcoma; and the depth of infiltration; mitotic figures (MF)/10 high power fields (HPF), counting in randomly selected areas of both components. In addition, we graded all sarcomas using the Trojani grading system. This grading system uses the parameters of tumour differentiation, necrosis, and mitotic activity. ${ }^{17}$

Immunohistochemical analysis was also performed. The dewaxed sections were incubated at room temperature for 30 minutes with the following antibodies: anti-vimentin (clone V6; DakoCytomation, Glostrup, Denmark; diluted 1/40), anti$\alpha$ smooth muscle actin (clone $\alpha \mathrm{sml}$; Novocastra, Newcastle upon Tyne, UK; diluted 1/50), anti-desmin (clone D33; DakoCytomation; diluted 1/40), anti-CD34 (clone QBEnd/ 10; Novocastra; diluted 1/25), and anti-factor XIIIa (FXIIIa; polyclonal; Calbiochem, San Diego, California, USA; diluted 1/50). We used a high pressure antigen retrieval method with citrate buffer and then a standard labelled streptavidinbiotin method with VIP (SK 4600; Vector Laboratories, Burlingame, California, USA) chromogene. CD34 and factor XIIIa immunoreactivity were graded semiquantitatively on a scale from 0 to $3+$.

Clinical details and follow up data were obtained from hospital records, referring pathologists, and clinicians.

\section{Control group}

We selected 20 cases of conventional DFSP from the remaining 79 cases, the criterion for selection being treatment by wide local excision. Formalin fixed, paraffin wax embedded material was available for evaluation. Paraffin wax embedded sections from all cases were processed routinely and stained with haematoxylin and eosin. The slides of each specimen were reviewed and confirmed by the authors. All tumours consisted of conventional DFSP without sarcomatous change. We recorded the clinical details of the patients and the maximum diameter of the tumours.

\section{RESULTS}

\section{Clinical features of transformed DFSP}

Table 1 lists the clinicopathological features of our patients. We examined five patients with DFSP-FS and three with DFSP-MFH. There were six women and two men and their age at diagnosis ranged from 26 to 62 years (median, 41). Most patients had a history of a superficial, slowly growing, painless mass; unusual clinical signs were not detected. The lesions were located on the trunk (six cases) and the lower extremities (two cases). Tumour size ranged from 3.5 to $8 \mathrm{~cm}$ (median, 4). After diagnosis, six of the patients were treated by wide local excision (cases 1, 2, 4, 5, 7, and 8) and two by incomplete local excision and radiotherapy, with or without chemotherapy. Surgical margins were positive in the last two cases (cases 3 and 6). (A positive margin was defined as tumour tissue on the inked margin of the surgical specimen.)

Follow up ranged from four to 36 years after the first operation, and in seven of eight cases it was more than five years.

Recurrences occurred in three of the eight cases: in two patients with positive surgical margins and in one patient who was treated by wide local excision. The overall

Table 2 Clinical features of 20 patients with DFSP treated by wide local excision (control group)

\begin{tabular}{|c|c|c|c|c|c|c|c|}
\hline Case & Age/sex & Site & $\begin{array}{l}\text { Tumour size } \\
(\mathrm{cm})\end{array}$ & Recurrence & $\begin{array}{l}\text { Interval to recurrence* } \\
\text { (no. of recurrences) }\end{array}$ & Metastasis & Follow up \\
\hline 1 & $28 / F$ & Back & 3 & - & - & - & NED; 4 years \\
\hline 2 & $34 / F$ & Neck & 2.5 & - & - & - & NED; 10 yrs \\
\hline 3 & $45 / M$ & Chest wall & 4 & - & - & - & NED; 8 years \\
\hline 4. & $41 / F$ & Trunk & 5 & + & 14 months (1) & - & NED; 12 years \\
\hline 5 & $33 / M$ & Leg & 3 & - & - & - & NED; 11 years \\
\hline 6 & $45 / M$ & Trunk & 3.5 & - & - & - & NED; 3 years \\
\hline 7 & $37 / F$ & Back & 2.4 & - & - & - & NED; 6 years \\
\hline 8 & $24 / F$ & Trunk & 4 & - & - & - & NED; 5 years \\
\hline 9 & $48 / M$ & Leg & 3.3 & - & - & - & NED; 8 years \\
\hline 10 & $35 / F$ & Upper extremity & 4 & - & - & - & NED; 7 years \\
\hline 11 & $28 / F$ & Shoulder & 5 & - & - & - & NED; 10 years \\
\hline 12 & $24 / F$ & Scalp & 2.8 & + & 24 months (1) & - & NED; 9 years \\
\hline 13 & $36 / F$ & Inguinal & 2 & - & - & - & NED; 6 years \\
\hline 14 & $42 / M$ & Trunk & 4 & + & 18 months (1) & - & NED; 7 years \\
\hline 15 & $23 / F$ & Back & 3.7 & - & - & - & NED; 14 years \\
\hline 16 & $37 / F$ & Neck & 3.4 & - & - & - & NED; 8 years \\
\hline 17 & $32 / M$ & Back & 2.8 & - & - & - & NED; 10 years \\
\hline 18 & $30 / M$ & Thigh & 4.6 & - & - & - & NED; 3 years \\
\hline 19 & $39 / F$ & Abdominal wall & 3.8 & - & - & - & NED; 7 years \\
\hline 20 & $40 / F$ & Shoulder & 4.9 & & & - & NED; 6 years \\
\hline
\end{tabular}


Table 3 Histopathological features of eight patients with DFSP-FS and DFSP-MFH

\begin{tabular}{|c|c|c|c|c|c|c|c|c|}
\hline Case & $\begin{array}{l}\text { Tumour phase } \\
\text { (presentation) }\end{array}$ & $\begin{array}{l}\text { Size of } \\
\text { sarcomatous } \\
\text { component }(\mathrm{cm})\end{array}$ & $\begin{array}{l}\text { Depth of } \\
\text { invasion }(\mathrm{cm})\end{array}$ & $\begin{array}{l}\text { Grade of } \\
\text { sarcomatous } \\
\text { component } \\
\text { (Trojani) }\end{array}$ & $\begin{array}{l}\text { Sarcomatous } \\
\text { component (\%) }\end{array}$ & $\begin{array}{l}\text { DFSP } \\
\text { component (\%) }\end{array}$ & $\begin{array}{l}\mathrm{MF} / 10 \mathrm{HPF} \\
\text { (DFSP) }\end{array}$ & $\begin{array}{l}\text { MF/10 HPF } \\
\text { (sarcoma) }\end{array}$ \\
\hline 1 & Original & $2 \times 2.5$ & 2.1 & 1 & 40 & 60 & 1 & 9 \\
\hline 2 & Original & $2.4 \times 2.9$ & 2.2 & 2 & 45 & 65 & 2 & 11 \\
\hline \multirow[t]{3}{*}{3} & Original & $2 \times 3$ & 4 & 2 & 25 & 75 & 1 & 10 \\
\hline & 1st recurrence & $2 \times 1$ & 3 & 2 & 45 & 55 & 2 & 11 \\
\hline & 2 nd recurrence & $2 \times 1$ & 3 & 2 & 40 & 60 & $\overline{1}$ & 12 \\
\hline \multirow[t]{2}{*}{4} & Original & $3 \times 2$ & 3 & 2 & 50 & 50 & 3 & 14 \\
\hline & 1st recurrence & $3 \times 3$ & 3.5 & 2 & 30 & 70 & 2 & 11 \\
\hline 5 & Original & $2 \times 2.1$ & 2 & 2 & 45 & 55 & 2 & 11 \\
\hline \multirow[t]{4}{*}{6} & Original & $3 \times 4$ & 3.5 & 2 & 35 & 65 & 2 & 13 \\
\hline & 1st recurrence & $3 \times 3$ & 3.2 & 2 & 45 & 55 & 3 & 12 \\
\hline & 2nd recurrence & $3 \times 2$ & 3 & 2 & 55 & 45 & 2 & 14 \\
\hline & 3rd recurrence & $2.8 \times 2$ & 2.2 & 2 & 50 & 50 & 2 & 16 \\
\hline 7 & Original & $3 \times 3$ & 3 & 2 & 70 & 30 & 2 & 9 \\
\hline 8 & Original & $1.5 \times 2$ & 2.4 & 2 & 45 & 65 & 2 & 11 \\
\hline
\end{tabular}

recurrence rate in sarcomatous variants was $37.5 \%$, but this was only $17 \%$ in patients who were treated by wide local excision.

The interval to recurrence ranged from nine months to four years. One patient developed three recurrences (case 6), two recurrences occurred in one patient (case 3), and one recurrence only was seen in one patient (case 4).

There were recurrences in two of the three DFSP-MFHs (recurrence rate of 66\%) and the surgical margins were positive in recurrent cases.

Recurrence was seen in one of the five patients with DFSPFS (recurrence rate of 17\%). The patient was treated by wide local excision with negative margins.

Case 3 was a 56 year old man who had noticed a $6 \times 4 \mathrm{~cm}$ mass in his left crural region. The lesion was excised and was diagnosed as neurilemmoma with sarcomatous change. The surgical margin was positive and the patient received postoperative irradiation without surgical re-excision. Four years later, a small recurrent nodule was found at the site of the previous one. A $2.5 \times 1.5 \mathrm{~cm}$ nodule was removed and a histological diagnosis of DFSP-MFH was made. The first diagnosis was deemed to be incorrect and we rediagnosed the tumour as DFSP-MFH. The surgical excision was incomplete and the margins of the second specimen were again positive. The patient did not receive postoperative irradiation or surgical re-excision after the second operation. Nine months later, a $2 \times 1.5 \mathrm{~cm}$ nodule was removed using wide local excision and the histological diagnosis was DFSP-MFH again. The surgical margins were negative. The patient showed no evidence of disease until he died from a heart attack 17 years after the primary operation. A necropsy was not performed.

Case 4 was a 41 year old woman who presented with a slowly growing mass on her trunk, which was removed. Histopathological examination diagnosed a DFSP-FS. The patient was treated by wide local excision, but 16 months later a small recurrent nodule was found at the site of the previous operation. The lesion was excised (wide local excision was used again) and the histopathological diagnosis was DFSP-FS. The patient has shown no evidence of disease since the second operation.

Case 6 was a 62 year old man who presented with a slowly growing mass on his trunk, which was excised. The histopathological diagnosis was DFSP-MFH with positive surgical margins. Three recurrences followed the primary operation (nine months, four years, and seven years after the primary excision), and the surgical margins were always positive except for on the last occasion. The patient received postoperative irradiation three times and additional che- motherapy once. After 4.5 years an enlarged lymph node was detected in the left inguinal area. Excision was again attempted and a diagnosis of metastatic MFH was made. The patient died from hypertension induced brain haemorrhage 13 years after the primary excision of DFSP-MFH. Clinical and radiological examinations excluded metastatic, haemorrhagic brain tumour, and generalised metastatic disease. A necropsy was not performed.

\section{Control group}

Table 2 summarises the clinicopathological data of the control group.

We examined 20 cases of conventional DFSP. The patients comprised 13 women and seven men and their ages ranged from 23 to 48 years (median, 35). The lesions were located on the trunk (10 cases), the lower extremities (three cases), the head and neck region (three cases), the shoulder region (two cases), the inguinal region (one case), and the upper extremity (one case). Most patients had a history of a superficial, slowly growing, painless mass. Tumour sizes ranged from 2 to $5 \mathrm{~cm}$ (median, 3.585). After the diagnosis, all patients were treated by wide local excision. Surgical margins were negative in all cases.

There were recurrences in three of the 20 cases (recurrence rate of $15 \%$ ). Recurrence occurred between 14 and 18 months after the primary surgical intervention (mean, 19).

The follow up interval ranged between three and 14 years (mean, 8.6).

\section{Histopathology}

The soft tissue tumour diagnoses were made according to the description of Weiss and Goldblum. ${ }^{5} 18$

Table 3 summarises the pathological features of the entire group of patients with DFSP-FS and DFSP-MFH.

All tumours consisted of areas of ordinary DFSP composed of uniform spindle shaped tumour cells with slender nuclei arranged in a storiform growth pattern, with intercellular collagen deposition and small capillary blood vessels scattered throughout (fig 1). Characteristic honeycomb or lacelike infiltration into the underlying subcutaneous fat was seen at least focally. There was little nuclear pleiomorphism and low mitotic activity. Mitotic counts ranged from 1 to 3 MF/10 HPF (median, 1.94). Myxoid change was conspicuous in one case (case 3). The ratio of DFSP in the complex lesions varied between cases from $45 \%$ to $75 \%$ (median, $54 \%$ ).

Sarcomatous change was found de novo in all cases.

The FS areas of the tumour showed a fascicular (no longer storiform), and highly cellular histological pattern, frequently 

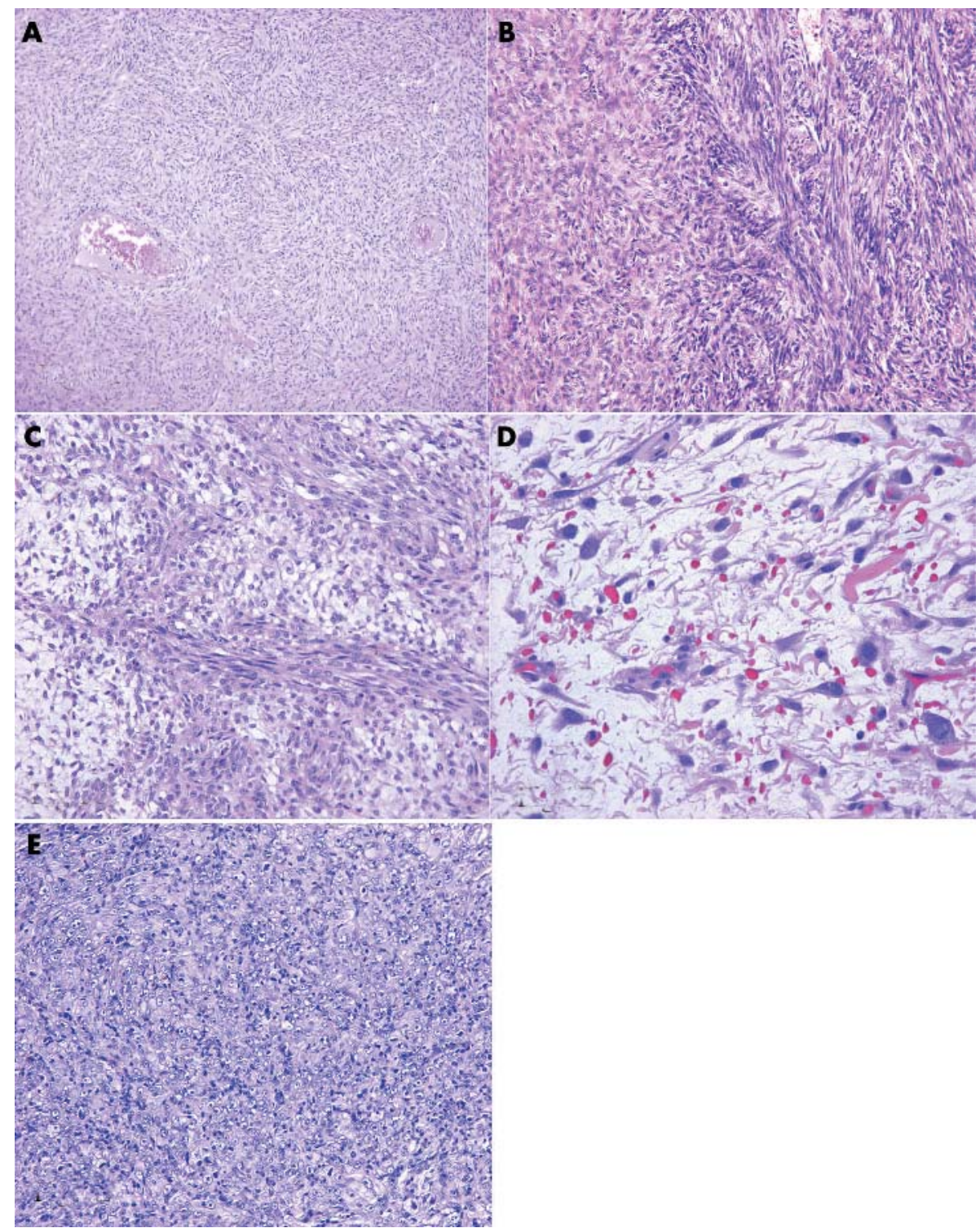

Figure 1 (A) All cases showed areas of typical dermatofibrosarcoma protuberans with a storiform growth pattern. (B) Fibrosarcomatous transformation of dermatofibrosarcoma protuberans; the transition to fibrosarcomatous area was gradual and subtle (case 2). (C); malignant fibrous histiocytoma (MFH) area of transformed dermatofibrosarcoma protuberans (DFSP) showing focal myxoid change with pleomorphic cellular features (case 3). (D) MFH area of transformed DFSP demonstrating atypical cellular features, and myxoid background (case 8). (E) Metastatic MFH showing undifferentiated cells, and pronounced pleomorphism (case 6).

exhibiting a characteristic herringbone pattern with the fascicles crossing at 45 degree angles. Tumour cells in the FS areas had slightly more plump nuclei than the tumour cells in areas of ordinary DFSP. FS areas showed moderate polymorphism and increased mitotic activity. Bizarre cellular features were not seen in FS. The FS areas were found in the subcutaneous part of the tumour, and the dermis was uninvolved in all cases. The interface between the DFSP and FS components was sharp in three cases and indistinct in two. Focal myxoid change of the FS areas was found in two of four cases.

In the MFH areas of the tumour the cells were large and pleomorphic. In these areas, multinucleated giant cells were common, and mitotic activity was increased. MFH areas were found in the subcutaneous part of the tumour, and the dermis was involved in two cases. The border between the DFSP and MFH components was indistinct in two cases and sharp in one. Focal myxoid change was found in two cases of MFH.
In general, proliferative activity was higher in sarcomatous areas. In areas of classic DFSP, the mitotic count ranged from 1 to $3 \mathrm{MF} / 10 \mathrm{HPF}$ (mean, 1.875), whereas in FS and MFH areas the mitotic counts ranged from 9 to $16 \mathrm{MF} / 10 \mathrm{HPF}$ (median, 12). In cases showing high proliferative activity in sarcomatous areas, neither local recurrence nor metastasis occurred.

Frank tumour necrosis and areas of extensive haemorrhage were seen in one case (case 3 ).

The metastatic tumour from patient 6 was composed exclusively of MFH without DFSP. The histopathological features of the metastatic tumour were identical to the original MFH described above.

The sarcomatous area was grade 1 in one case and grade 2 in seven cases.

\section{Immunohistochemistry}

Table 4 lists the immunohistochemical findings. 


\begin{tabular}{|c|c|c|c|c|c|c|c|c|}
\hline Case & $\begin{array}{l}\text { Tumour phase } \\
\text { (presentation) }\end{array}$ & $\begin{array}{l}\text { Vimentin (DFSP and } \\
\text { sarcoma) }\end{array}$ & CD34 (sarcoma) & CD34 (DFSP) & FXIIla (sarcoma) & FXIlla (DFSP) & Desmin & $\alpha$-SMA \\
\hline 1 & Original & + & - & ++ & - & + & - & - \\
\hline 2 & Original & + & + & +H+ & - & + & - & - \\
\hline \multirow[t]{3}{*}{3} & Original & + & - & ++ & ++ & + & - & - \\
\hline & 1 st recurrence & + & - & ++ & ++ & + & - & - \\
\hline & 2nd recurrence & + & - & ++t & +++ & ++ & - & - \\
\hline \multirow[t]{2}{*}{4} & Original & + & + & +H+ & - & + & - & - \\
\hline & 1 st recurrence & + & - & ++t & - & + & - & - \\
\hline 5 & Original & + & - & +H+ & - & + & - & - \\
\hline \multirow[t]{4}{*}{6} & Original & + & - & ++ & H+ & + & - & - \\
\hline & 1st recurrence & + & - & ++ & H+ & + & - & - \\
\hline & 2nd recurrence & + & - & ++ & H+ & ++ & - & - \\
\hline & 3rd recurrence & + & - & ++ & H & + & - & - \\
\hline 7 & Original & + & + & +++ & - & + & - & - \\
\hline 8 & Original & + & - & +H+ & - & + & - & - \\
\hline
\end{tabular}

Vimentin was positive in conventional DFSP and in the FS or MFH areas of all of cases. Tumour cells in conventional DFSP areas stained strongly for CD34 in all cases, whereas in FS areas only three cases were positive. There was no CD34 positivity in MFH areas. Factor XIIIa reactivity was seen only in scattered spindle cells in the conventional DFSP areas of all cases, whereas we found a diffuse strong reaction in more than $2 \%$ of cells in MFH areas, but not in FS areas. All tumours were negative for desmin and $\alpha$ smooth muscle actin.

\section{DISCUSSION}

DFSP is a locally aggressive fibrohistiocytic neoplasm of intermediate malignancy. Although it is understood that DFSP has a potential for local recurrence and a low risk of distant metastasis, it has been repeatedly shown that the size of the resection directly influences the recurrence rate in DFSP. The rate of recurrence following wide local excision in conventional DFSP is $18 \%{ }^{18}$

In 1951, Penner reported a case of metastasising DFSP containing areas that were histologically indistinguishable from fibrosarcoma. ${ }^{6}$ More recently, Ding et al suggested a higher recurrence rate in cases of FS transformation of DFSP, but the surgical treatments were not well described in their report. ${ }^{7}$ Similar observations were documented by Mentzel et al and Pizarro et al, but in their studies either only a minority of patients received wide local excision, which is the standard treatment for DFSP, or treatment details were not provided. ${ }^{13}{ }^{14}$

In contrast, $\mathrm{O}^{\prime}$ Conell and Trotter reported that recurrences were dependent on the adequacy of resection. ${ }^{12}$ One of the largest studies by Goldblum et al found no prognostic differences between conventional DFSP and DFSP containing sarcoma treated by wide local excision. ${ }^{16}$

In our study, we evaluated the clinicopathological profile of conventional DFSP and DFSP containing sarcoma (FS or $\mathrm{MFH}$ ), with an emphasis on the longterm follow up. Sarcomatous change occurred in eight of the 87 DFSP cases in our study. We analysed eight patients with sarcomas arising in DFSP (five patients with DFSP-FS and three with DFSP-MFH) and 20 patient with conventional DFSP. There were no remarkable differences in age and anatomical site between the patients with DFSP containing sarcoma and those with ordinary DFSP. The sarcomatous change presented in de novo cases, and not as recurrences. All conventional DFSPs were treated by wide local excision, and the recurrence rate was found to be $15 \%$, which is lower than the published rate.

For the clinicopathological correlations, we chose to study six patients with DFSP containing sarcoma, who were treated by wide local excision, and two other patients with transformed DFSP who were treated by incomplete local excision(s) and additional radiotherapy, with or without chemotherapy. In all of our transformed cases the sarcomatous area constituted a significant proportion of the tumour, and ranged from $25 \%$ to $70 \%$. The length of our follow up period was sufficient to encompass the initial three year period, during which most recurrences occur. ${ }^{13}{ }^{16}$

\section{"Dermatofibrosarcoma protuberans with sarcomatous foci is a more aggressive tumour, which had a $12.5 \%$ metastatic rate in our study"}

In our study, there were recurrences in three cases of transformed DFSP, and the total recurrence rate of DFSP-FS and DFSP-MFH was $37.5 \%$. The total recurrence rate of DFSP containing sarcoma in our study was significantly higher than that of our control group. Unfortunately, only one patient with recurrent DFSP containing sarcoma was treated with wide local excision. When the treatment was simple local excision, the recurrence rate was $100 \%$. Five patients were free of local recurrences and/or metastases. The metastasis seen in one patient with DFSP-MFH could be attributed to the aggressive nature of the transformed tumour. The overall recurrence rate of DFSP containing sarcoma was $17 \%$ when treated by wide local excision. This observation indicates that DFSPs with sarcoma that lack positive surgical margins may have the same clinical behaviour in longterm follow up as conventional DFSP treated by wide local excision, but DFSP with sarcomatous foci is a more aggressive tumour, which had a $12.5 \%$ metastatic rate in our study. However, the metastatic rate in conventional DFSP is less than $3 \%$.

The immunohistochemical demonstration of CD34 is an important feature for diagnosing DFSP. ${ }^{45}$ In all our cases of conventional DFSP, areas of strong CD34 positivity were noted, whereas the sarcomatous areas showed weak CD34 reactivity in three of eight cases. This can be explained by loss of expression of CD34 during tumour dedifferentiation.

Recurrence and metastatic potential were not influenced by the depth of invasion, the amount of sarcomatous area, or the histological grade of the sarcomatous component. Nevertheless, more cases should be studied to make a definite statement about the factors affecting the outcome of DFSP containing sarcomatous change.

Local treatment is a crucial determinant of the behaviour of tumours in general, and in the case of low or high grade extremity sarcomas. ${ }^{19}$ The recurrence potential of DFSP is directly related to the extent of resection. Micrographical 


\section{Take home messages}

- Sarcomatous change in dermatofibrosarcoma protuberans (DFSP) is a form of tumour progression, and is associated with a worse prognosis than ordinary DFSP

- The degree of aggression of DFSP containing sarcomas may be related to the histological grade of sarcomatous transformation

- Although transformed DFSP is more aggressive, prognosis is influenced by the extent of excision, and with wide excision there may be little increased risk for recurrence and metastasis over that of conventional DFSP

surgical resection could theoretically reduce the risk of recurrence to zero, ${ }^{20}$ although it can be very difficult to determine a positive margin because DFSP has fibroblast-like morphology. In the absence of recurrence, the risk of metastasis is greatly reduced, because metastasis never occurs without antecedent local recurrence. Thus, in our opinion, wide local excision with negative margins reduces and sometimes eradicates the risk of local recurrence or dissemination.

However, the tumours that are removed by wide local excision with clear margins probably represent a less aggressive subset of lesions, as shown in high grade extremity sarcomas. ${ }^{19}$

In summary, we have shown that sarcomatous change in DFSP represents a form of tumour progression, and is associated with a worse prognosis than ordinary DFSP. The degree of aggression of DFSP containing sarcomas may be related to the histological grade of sarcomatous transformation. Even though transformed DFSP is a more aggressive tumour, the prognosis can be influenced by the extent of excision, so that there may be little increased risk for relapse and dissemination over that of conventional DFSP.

\section{Authors' affiliations}

Z Szollosi, Z Nemes, Department of Pathology, University of Debrecen, Medical and Health Science Centre, Debrecen, H-4012 Hungary

\section{REFERENCES}

1 Taylor HB, Helwig EB. Dermatofibrosarcoma protuberans. A study of 115 cases. Cancer 1962;15:717-25.

2 McPeak CJ, Cruz T, Nicastri AD. Dermatofibrosarcoma protuberans: an analysis of 86 cases - five with metastasis. Ann Surg 1967;166:803-16.

3 Taylor HB, Helwig EB. Dermatofibrosarcoma protuberans: study of 115 cases. Cancer 1962;15:717-25.

4 Fletcher CDM, Evans BJ, Macartney JC, et al. Dermatofibrosarcoma protuberans: a clinicopathological and immunohistochemical study with a review of the literature. Histopathology 1985;9:921-38.

5 Weiss SW, Goldblum JR. Fibrohistiocytic tumors of intermediate malignancy. In: Weiss SW, Goldblum JR, eds. Enzinger and Weiss's soft tissue tumors, 4th ed. St Louis: Mosby, 2001:491-534.

6 Penner DW. Metastasizing dermatofibrosarcoma protuberans: a case report. Cancer 1951;4:1083-6.

7 Ding J, Hashimoto H, Enjoji M. Dermatofibrosarcoma protuberans with fibrosarcomatous areas. A clinicopathologic study of nine cases and a comparison with allied tumors. Cancer 1989;64:721-9.

8 Conelly JH, Evans HL. Dermatofibrosarcoma protuberans. A clinicopathologic review with emphasis on fibrosarcomatous areas. Am J Surg Pathol 1992; 16:921-5.

9 Calonje E, Fletcher CDM. Myoid differentiation in dermatofibrosarcoma protuberans and its fibrosarcomatous variant: clinicopathologic analysis of 5 cases. J Cutan Pathol 1996;23:30-6.

10 Diaz-Cascajo C, Weyers W, Borrego L, et al. Dermatofibrosarcoma protuberans with fibrosarcomatous areas: a clinico-pathologic and immunohistochemical study in four cases. Am J Dermatopathol 1997; 19:562-7.

11 Wrotnowski U, Cooper PH, Shmookler BJ. Fibrosarcomatous change in dermatofibrosarcoma protuberans. Am J Surg Pathol 1998;12:287-93.

12 O'Conell JX, Trotter MJ. Fibrosarcomatous dermatofibrosarcoma protuberans: a variant. Mod Pathol 1996;9:273-8.

13 Mentzel T, Beham A, Katenkamp D, et al. Fibrosarcomatous ("high- grade") dermatofibrosarcoma protuberans. Clinicopathologic and immunohistochemical study of a series of 41 cases with emphasis on prognostic significance. Am J Pathol 1998;22:576-87.

14 Pizarro GB, Fanburg JC, Miettinen M. Dermatofibrosarcoma protuberans with fibrosarcomatous transformation: re-explored [abstract]. Mod Pathol 1997; 10:55A.

15 Dowd JO, Laidler P. Progression of dermatofibrosarcoma protuberans to malignant fibrous histiocytoma: report of a case with implications for tumor histogenesis. Hum Pathol 1998;19:368-70.

16 Goldblum JR, Reith JD, Weiss SW. Sarcomas arising in dermatofibrosarcoma protuberans. A reappraisal of biologic behaviour in eighteen cases treated by wide local excision with extended clinical follow up. Am J Pathol 2000;24: $1125-30$

17 Coindre JM, Nguyen BB, Bonichon F, et al. Histopathologic grading in spindle cell soft tissue sarcomas. Cancer 1988;61:2305-9.

18 Weiss SW, Goldblum JR. Malignant fibrohistiocytic tumors. In: Weiss SW, Goldblum JR, eds. Enzinger and Weiss's soft tissue fumors, 4th ed. St Lovis: Mosby, 2001:535-70.

19 Gaynor JJ, Tan CC, Casper ES, et al. Refinement of clinicopathologic staging for localized soft tissue sarcoma of the extremity: a study of 423 adults. J Clin Oncol 1992;10:1317-29.

20 Gloster HM, Harris KR, Roenigk RK. A comparison between Mohs micrographic surgery and wide local excision for the treatment of dermatofibrosarcoma protuberans. J Am Acad Dermatol 1996;35:82-7. 